\title{
Tratamiento quirúrgico de la escoliosis idiopática del adolescente. Resultados en el Instituto Nacional de Rehabilitación de México. Seguimiento mínimo de 24 meses
}

\author{
Idiopathic scoliosis's surgical treatment. Results in Mexico's National Rehabilitation \\ Institute. 24 months follow
}

\author{
Barón Zárate-Kalfópulos ${ }^{1 *}$, Héctor R. Martínez-Ríos², Francisco López-Meléndez, \\ Carla L. García-Ramos ${ }^{1}$, Luis M. Rosales-Olivarez y Alejandro A. Reyes-Sánchez ${ }^{1}$ \\ ${ }^{1}$ División de Cirugía de Columna Vertebral; '2División de Ortopedia. Instituto Nacional de Rehabilitación, Ciudad de México, México
}

\begin{abstract}
Resumen
Antecedentes: La escoliosis idiopática del adolescente se define como una deformidad tridimensional de la columna vertebral que se presenta entre los 10 y los 18 años, y que se manifiesta con una curvatura vertebral en el plano coronal mayor de $10^{\circ}$. Esta deformidad afecta al 2-3\% de la población general, pero solo el $10 \%$ del total requerirá en algún momento tratamiento quirúrgico. El método de elección para el manejo es el uso de tornillos transpediculares y barras desrotadoras. Objetivo: Realizar un análisis descriptivo de los pacientes que recibieron manejo quirúrgico en nuestro instituto con tornillos transpediculares y barras. Método: Se trata de un estudio observacional, retrospectivo, analítico, abierto, de muestreo no probabilístico, en el que se incluyeron los pacientes tratados con manejo quirúrgico entre 2012 y 2013. Las deformidades se estratificaron de acuerdo con la clasificación de Lenke. El ángulo de corrección de la deformidad, los niveles instrumentados, el sangrado transquirúrgico y la presencia de complicaciones fueron las variables analizadas. Resultados: La mayoría de los pacientes presentaron curvas Lenke IBN, Nash Moe III, Cobb un promedio de 59.4 y cifosis de 47.8. En promedio se siguió a los pacientes por 35.84 meses, detectando un aumento de la curvatura coronal de $2.28^{\circ}$ y un aumento de la curvatura sagital de 2.8 . Conclusión: Al comparar estos resultados y la literatura mundial se concluyó que el tratamiento de la escoliosis idiopática del adolescente es un método seguro y reproducible que ofrece una mayor ventaja biomecánica y biológica sobre el uso de instrumentación mixta utilizada anteriormente.
\end{abstract}

PALABRAS CLAVE: Escoliosis idiopática del adolescente. Tornillos transpediculares. Artrodesis de columna vertebral. Resultados de tratamiento.

\begin{abstract}
Background: Adolescent's idiopathic scoliosis is defined as a three-dimensional deformity of the spine, which occurs between 10 and 18-year-old, has a spinal curvature $>10^{\circ}$ in the coronal plane. This deformity affects $2-3 \%$ of the general population, however, only $10 \%$ of the total will require surgery at some point. The method of choice for management is the use of pedicle screws and rods derotational. Objective: To perform a descriptive analysis of patients who received surgical treatment in our institute with pedicle screws and rods. Methods: This is an observational, retrospective, analytical, open study, non-probabi-
\end{abstract}

\author{
Correspondencia: \\ *Barón Zárate-Kalfópulos \\ Calzada México-Xochimilco 289 \\ Col. Arenal de Guadalupe, Del. Tlalpan \\ C.P.14389, Ciudad de México, México \\ E-mail: baronzk@ @otmail.com
}

Fecha de recepción: 04-12-2017

Fecha de aceptación: 16-06-2018

DOI: 10.24875/CIRU.18000012
Cir Cir. 2018;86:392-398

Contents available at PubMed www.cirugiaycirujanos.com 
lity sampling, in which patients requiring surgical treatment at our institute between 2012 and 2013 were included, the deformities were stratified according to the classification of Lenke. The angle of deformity correction, instrumented levels, amount of bleeding, presence of complications were the variables analyzed. Results: Lenke classifying mostly IBN, Moe Nash III, an average of $59.4^{\circ} \mathrm{Cobb}$ and kyphosis of 47.8 . On average it was followed patients for 35.84 months, detecting an increase $2.28^{\circ}$ coronal curvature and sagittal curvature increase of $2.8^{\circ}$. Conclusion: Comparing these results and world literature concluded that the treatment of adolescent's idiopathic scoliosis is a safe and reproducible method that provides greater biomechanical and biological advantage over the use of mixed instrumentation used previously.

KEY WORDS: Adolescent idiopathic scoliosis. Pedicle screws. Spinal fusion. Treatment outcome.

\section{Introducción}

La escoliosis idiopática del adolescente (EIA) es una deformidad de la columna vertebral en el plano coronal, axial y sagital, con un curvatura en el plano coronal mayor de $10^{\circ}$, que afecta a pacientes entre 10 y 18 años, aquejando el estado psicológico, funcional y cosmético'.

La presentación clásica ( $80 \%$ de la población) es la de un paciente mujer con un curvatura torácica mayor de $20^{\circ}$, deformidad rotacional e hipocifosis ${ }^{1}$. Es una patología relativamente común, que afecta al 2-3\% de la población general, pero solo el 10\% requerirá en algún momento tratamiento quirúrgico².

De las teorías descritas, el factor genético es el que se ha asociado como probable origen de esta patología, sin ser concluyente ${ }^{3}$. Debido a la gran variabilidad de presentación, influida por factores como el sexo, la edad de aparición, el pico de crecimiento esquelético, el tamaño y el tipo de curva, se dificultan su descripción y estudio".

King fue el primero en realizar una clasificación anatomomorfológica de las deformidades torácicas, pero no tomó en cuenta las curvaturas lumbares, y además estudios subsecuentes reportan una alta variabilidad interobservador e intraobservador ${ }^{4,5}$. La clasificación más utilizada en la actualidad es la descrita por Lenke, diseñada para la elaboración de la planeación quirúrgica. Con esta clasificación se reportan menores limitantes que con la clasificación de King y es útil para comparar diferentes tratamientos o tratamiento únicos, presenta menor variabilidad intraobservador e interobservador, y brinda mayor seguridad en la realización de un plan quirúrgico ${ }^{6}$.

Si la deformidad vertebral tiene una curvatura mayor de $50^{\circ}$, la función respiratoria y cardiaca se ven afectadas, y el paciente reporta dolor crónico y fatiga para realizar las actividades de la vida diaria?.

Las indicaciones quirúrgicas de la EIA son una curvatura mayor de $50^{\circ}$ en el plano coronal, una curvatura mayor de $40^{\circ}$ en un paciente con inmadurez esquelética, la progresión de la enfermedad a pesar del tratamiento conservador con el uso de corsé y una deformidad inaceptable por el paciente ${ }^{8}$.

Los objetivos del tratamiento quirúrgico con tornillos transpediculares y barras son obtener una corrección significativa, funcional y estética, lograr una artrodesis vertebral estable, menores tasas de no unión (permite realizar osteotomías), prevenir la recurrencia y la pérdida de la corrección, y permitir al paciente una movilidad posoperatoria temprana'.

En 1962, Harrington describió el uso de barras de distracción para el manejo de la escoliosis, y reportó poco control de la corrección sagital de la deformidad $^{9-11}$. En los años 1980 se introdujo el uso de ganchos y alambres sublaminares por Cotrel Dobousset y Luque, respectivamente; estos sistemas permitieron un mejora en el control de la corrección sagital y coronal, además de una movilización temprana del paciente ${ }^{10,12}$.

En 1963, Roy-Camille introduce los tornillos transpediculares con el objetivo de tratar las patologías localizadas únicamente en la región lumbar ${ }^{10,13}$. Sin embargo, su uso fue recomendado solo bajo circunstancias específicas, por el peligro (lesión vascular, nerviosa y visceral) y la dificultad que representaba la colocación de los tornillos debido a la complicada morfología pedicular, que sufre una alteración anatómica en volumen (variando de 2.5 a $12 \mathrm{~mm}$ en anchura y de 9.6 a $16 \mathrm{~mm}$ en altura) y dirección (orientación en el plano axial, sagital y coronal), afectada por el nivel vertebral, la edad y la talla del paciente ${ }^{1,5}$. Para disminuir la dificultad en la colocación de los tornillos transpediculares se toman en cuenta factores que aumentan la seguridad, como el factor anatómico estructural, la pared medial pedicular que es más fuerte que la pared lateral ${ }^{14}$. Gerztbein y Robbins ${ }^{15}$ describieron la zona de seguridad, que abarca $4 \mathrm{~mm}$ añadidos al borde medial del pedículo, que corresponde a $2 \mathrm{~mm}$ de espacio epidural y $2 \mathrm{~mm}$ de espacio subaracnoideo. 
Se usó este mismo concepto en la colocación de ganchos sublaminares, ya que estos abarcan un área de 2-3 $\mathrm{mm}$ de esta zona de seguridad ${ }^{16}$. Sin embargo, el objetivo de la descripción de la zona de seguridad no es utilizarla en su totalidad, ya que la colocación es aceptable cuando el tornillo rebasa en $2 \mathrm{~mm}$ la cortical medial ${ }^{1,16}$.

Actualmente, los sistemas de ganchos y alambres están en desuso debido a la implementación y la posterior demostración de las ventajas del tratamiento con tornillos transpediculares y barras ${ }^{1}$. La colocación de tornillos transpediculares y barras corrige la deformidad en las tres columnas anatómicas, previniendo el efecto cigüeñal, disminuyendo el número de niveles instrumentados y ofreciendo una mejor corrección de la deformidad ${ }^{1,17}$ (Fig. 1).

Suk et al. ${ }^{17}$ son pioneros en el uso de tornillos transpediculares en todos los pedículos, demostrando su seguridad en el tratamiento de la EIA. Reportaron sus resultados con un estudio en el que incluyeron 462 pacientes (colocando 4604 tornillos) y obtuvieron una mala posición en 48 pacientes $(1.5 \%$ del total de tornillos), de los cuales solo cuatro tornillos rebasaron la cortical medial, sin reportar ninguna lesión neurológica, vascular ni visceral. Demostraron que, bajo el entrenamiento y la experiencia adecuados, la colocación de los tornillos no es influenciada por la deformidad vertebral global. Kuklo et al. ${ }^{4}$ reportaron la colocación de tornillos en 20 pacientes con una curvatura global de $100.2^{\circ}$, con una precisión del $96.3 \%$ en el posicionamiento de los tornillos, sin reportar lesiones neurovasculares ni viscerales.

La evolución al tratamiento de la EIA con tornillos transpediculares y barras demuestra una superioridad biomecánica, con menores tasas de complicaciones, mejorando la corrección de la deformidad radiográfica y clínica del paciente, en comparación con las técnicas usadas anteriormente en nuestro instituto'.

A la fecha, en México no existen estudios descriptivos que nos permitan conocer los resultados radiográficos, clínicos y funcionales del tratamiento de la EIA con tornillos transpediculares y barras desrotadoras. Se busca conocer la casuística de pacientes con EIA tratados de forma quirúrgica, la prevalencia de complicaciones, comparar los resultados obtenidos con técnicas realizadas previamente en nuestro instituto y en otros centros especializados, conocer el porcentaje de corrección, y describir y analizar otras variables (sangrado, tiempo quirúrgico y niveles instrumentados).

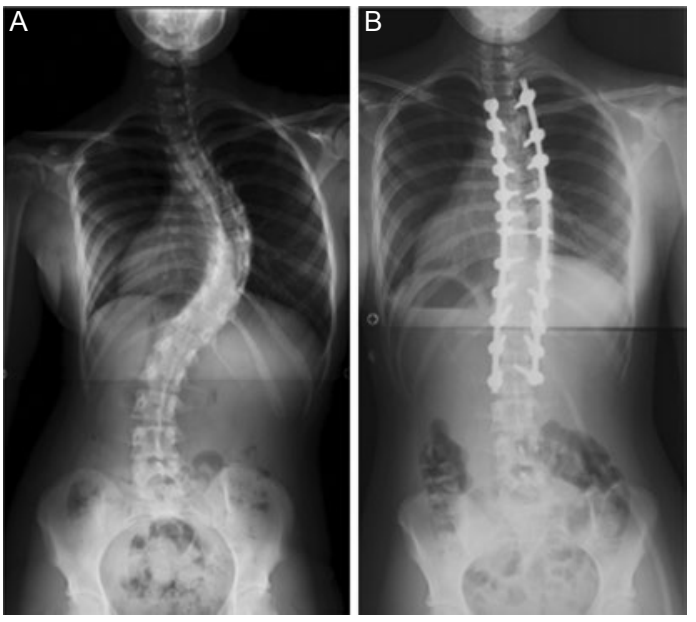

Figura 1. Radiografías anteroposteriores, prequirúrgica $(\boldsymbol{A})$ y posquirúrgica (B), de un paciente con escoliosis idiopática del adolescente. Se observa una corrección de $54^{\circ}$ posquirúrgica en el plano coronal con el uso de tornillos transpediculares.

\section{Método}

Se analizaron los expedientes de pacientes de entre 13 y 18 años con diagnóstico de EIA tratados mediante manejo quirúrgico con tornillos pediculares y barras desrotadoras, operados en nuestro instituto en el periodo comprendido entre 2010 y 2013, con seguimiento mínimo de 2 años, que tuvieran expediente clínico e imagenológico completo.

La recolección de datos se llevó a cabo por dos ortopedistas que no se involucraron directamente con los procedimientos quirúrgicos de los pacientes. Los datos estadísticos fueron analizados con el programa SPSS (versión 21). Se realizó análisis con estadística descriptiva para ver frecuencias, porcentajes, medias y desviaciones estándar. Se realizaron una prueba de Kolmogorov-Smirnov para verificar la normalidad de la muestra, y una prueba t para muestras apareadas (relacionadas) para variables continuas, para ver diferencias. Se hizo una prueba de análisis de varianza (ANOVA) para ver diferencias de corrección entre los distintos grupos de la clasificación de Lenke en cuanto al porcentaje de corrección. Todas las pruebas estadísticas fueron cruzadas y se consideró estadísticamente significativo un valor de $p<0.05$, con su intervalo de confianza (IC) del 95\%.

\section{Resultados}

Se obtuvieron 43 pacientes con diagnóstico de EIA, de los cuales 25 reunieron los criterios de inclusión para este estudio. Se incluyeron 2 hombres y 23 mujeres $(92 \%)$, con una distribución normal de edad de 
17 años ( \pm 2.79 años). Veintidós pacientes tenían una deformidad reducible y tres tenían una deformidad irreductible; solo dos pacientes presentaban un déficit neurológico (ASIA D). Se clasificaron, según Lenke, de acuerdo con el tipo de curva: curvatura tipo I en 14 pacientes (56\%), tipo III en 8 pacientes, tipo II en 4 pacientes y tipo VI en 2 pacientes. Según el modificador lumbar hubo 11 pacientes con un modificador lumbar B (44\%), 9 con un modificador lumbar C $(36 \%)$ y 5 con un modificador lumbar $A$. De acuerdo con el modificador sagital hubo 15 pacientes $(60 \%)$ con cifosis torácica a neutro $\left(10-40^{\circ}\right), 8$ pacientes con aumento de la cifosis (mayor de $40^{\circ}$ ) y 2 pacientes con disminución de la cifosis (menor de $10^{\circ}$ ). Se realizó la medición de la deformidad coronal con el método de Cobb, encontrando un promedio de $60.8^{\circ}$ con una oscilación de 38 a $113^{\circ}$ (desviación estándar: \pm 15.87 ) y una cifosis promedio de $47.8^{\circ}$ con una oscilación de 10 a $90^{\circ}$. El promedio de niveles afectados fue de 9.92 (rango: 6-14). También se clasificó la rotación de la vértebra ápice mediante la escala de Nash Moe, encontrando 14 con una deformidad tipo 3, 10 con una deformidad tipo 2, y 1 con una deformidad tipo 4.

Posterior a completar el protocolo prequirúrgico se sometieron los 25 pacientes al procedimiento quirúrgico. De ellos, 23 fueron abordados posteriormente $(92 \%)$ y 2 de manera anterior y posterior, con un sangrado de 350 a $4500 \mathrm{cc}$ (promedio de $1146 \mathrm{cc}$ ), un tiempo quirúrgico promedio de 320.72 minutos (oscilando de 210 a 480 minutos) e instrumentando en promedio 10.88 niveles (rango: 4-15).

Seis pacientes sufrieron complicaciones transquirúrgicas y en el posoperatorio inmediato (infección, hipovolemia, lesión dural, lesión medular, recorte de barra, lesión pleuropulmonar), pero solo tres requirieron cirugía de revisión (infección, tiempo quirúrgico prolongado). El promedio de estancia hospitalaria fue de 8.88 días ( \pm 7.06 días) (Fig. 2).

Tras el evento quirúrgico se realizaron estudios de imagen y se encontró un porcentaje promedio de reducción de la deformidad coronal del 64\% (21.6 \pm $\left.11.66^{\circ}\right)$ y de la deformidad sagital del $66.71 \%(33.7 \pm$ $\left.16.6^{\circ}\right)$, con un promedio de corrección rotacional Nash Moe de 2.

Se siguió a los pacientes al menos por 24 meses (promedio de $35.84 \pm 11.4$ meses) con interrogatorio, exploración física y estudios de imagen (radiografías anteroposterior y lateral de columna panorámica), observando un mínimo aumento de la curvatura coronal de $2.28^{\circ}\left(23.9 \pm 12.57^{\circ}\right)$. Se realizó una prueba t para

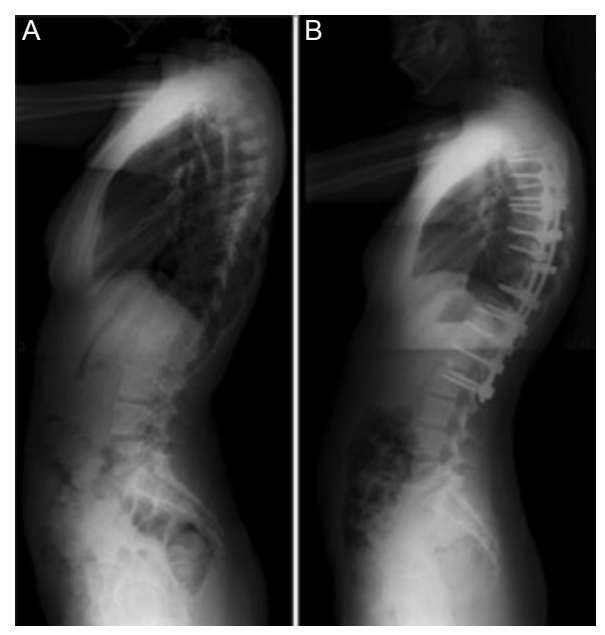

Figura 2. Radiografías laterales, prequirúrgica (A) y posquirúrgica (B), de un paciente con diagnóstico de escoliosis. Pueden verse los tornillos transpediculares en la columna toracolumbar.

muestras relacionadas para ver la significancia estadística, y se encontró una $p<0.0001$ en el ángulo de Cobb prequirúrgico y al final del seguimiento, sin hallar diferencia entre el posquirúrgico inmediato y el seguimiento $(p>0.05)$.

Se reportó un aumento de la curvatura sagital de $2.8^{\circ}$ (promedio de $35.84 \pm 16.69^{\circ}$ ) con una $p<0.001$; se encontró diferencia entre el posquirúrgico inmediato $y$ el final del seguimiento $(p=0.017)$.

Durante el seguimiento se reportó la rotura de la barra en un paciente, que hasta el momento del estudio no requirió cirugía de revisión. Se realizó a todos los pacientes el cuestionario SRS-22, en el que se registró un resultado promedio en los rubros de dolor de 2.8 , en autoimagen de 3.8, en función de 3 , en salud mental de 3 y en satisfacción quirúrgica de 3.5 , en una escala de 1 a 5.

\section{Discusión}

En la literatura existen pocos reportes con seguimiento a largo plazo del tratamiento de la EIA con tornillos transpediculares y barras, encontrando en la mayoría de las series con seguimiento no mayor de 24 meses una mínima pérdida de la reducción ${ }^{1}$.

Nuestra serie, compuesta en un $76 \%$ por pacientes de sexo femenino, concuerda con lo reportado por Westrick y Ward ${ }^{2}$, quienes publican una serie de 99 pacientes operados con la técnica mencionada con una incidencia del mismo sexo del $79 \%$. Una revisión epidemiológica concluye que la incidencia total de EIA (en pacientes operados y que recibieron tratamiento conservador) es mucho mayor en las mujeres que en los hombres ${ }^{8}$. 
Hwang et al. ${ }^{1}$, en el año 2012, realizaron un estudio multicéntrico y registraron el tipo de deformidad según Lenke, encontrando el $54.4 \%$ con tipo I, con un promedio de curvatura principal de $54.7^{\circ}$ y una cifosis de $22.3^{\circ}$ (medida de T5 a T12). En cambio, en nuestra documentación reportamos un $56 \%$ de deformidad coronal de tipo I, una curva principal promedio de $59.4^{\circ}$ y una cifosis de $47.8^{\circ}$, difiriendo en especial este último punto. Al analizar este resultado observamos cómo afecta el estudio de pequeño tamaño, ya que tener pacientes con una deformidad en cifosis de hasta $90^{\circ}$ desvía la media.

Estudiamos la rotación de la vértebra ápice mediante la escala de Nash Moe, reportando 14 pacientes con una deformidad tipo III, 10 con una deformidad tipo II y uno con una deformidad tipo IV. Kuklo et al. ${ }^{4}$ realizaron un estudio en el que incluyeron 19 pacientes Lenke I, Nash Moe III. A todos ellos se les realizó el estudio de imágenes radiográficas y se comparó contra el estudio de tomografía computada; en los 38 estudios se midió la rotación de la vértebra ápice mediante el medido de Nash Moe, observando una tendencia al aumento del puntaje Nash Moe con el estudio radiológico, mencionando que incluso el grado 0 determinado mediante una técnica radiográfica presenta algún tipo de rotación demostrada por tomografía computada. Sin embargo, el costo de realizar una tomografía computada solo para la medición de la rotación axial supera el beneficio, ya que expone al paciente a un alto grado de radiación y además supone un alto costo económico, sin afectar el plan prequirúrgico realizado con imágenes radiológicas y exploración física ${ }^{4}$.

Kim et al..$^{18}$ realizaron un estudio retrospectivo con 29 pacientes, en el que registraron un tiempo quirúrgico promedio de 347 minutos, 27 minutos más que el reportado en nuestro estudio (320.72 minutos). Registramos una pérdida sanguínea promedio de 1146 cc, contra una pérdida sanguínea reportada por Kim et al. ${ }^{18}$ de $961 \mathrm{cc}$, con una diferencia promedio de 185 cc. Al analizar la elevación del promedio de sangrado se observó que los pacientes con mayor volumen de pérdida sanguínea fueron aquellos con cirugías de mayor tiempo quirúrgico y, por lo tanto, mayor tiempo anestésico. Debido al pequeño tamaño de muestra, el aumento del volumen promedio del sangrado desvía nuestra media.

Lehman, et al. ${ }^{3}$ reportan una media de niveles fusionados de 10 , mientras que en nuestro estudio reportamos 9.92 niveles fusionados en promedio. Un estudio realizado en 2005 por Suk et al..$^{5}$ encontró que el uso de instrumentación con tornillos transpediculares disminuye el número de niveles fusionados comparado con el uso de fijación híbrida, con similitud de resultados.

En nuestra serie se reportó una tasa de complicaciones del $24 \%$, mientras que la tasa de complicaciones reportadas en la literatura mundial oscila del 4.4 al $51 \%{ }^{1,3}$. El paso de mayor importancia para evitar complicaciones es la colocación de los tornillos transpediculares; se requiere una larga curva de aprendizaje, incluso para un cirujano de gran experiencia $^{5}$, e influye de manera importante en la gran variabilidad en cuanto a las complicaciones reportadas, como lesión medular, sangrado, desanclaje y fracturas.

Suk, et al. ${ }^{5}$ realizaron un estudio con 203 pacientes con diagnóstico de EIA tratados con tornillos transpediculares y barras, y concluyen que este tipo de instrumentación demuestra una gran ventaja biomecánica sobre otras formas de instrumentación vertebral, al corregir y mantener la corrección realizada de la deformidad.

A los 2 años de seguimiento se encontró una pérdida de la reducción de la deformidad coronal de $2.28^{\circ}$ y una pérdida de la reducción de la curvatura sagital de $2.8^{\circ}$, comparado con un seguimiento a 2 años realizado por Hwang, et al. ${ }^{1}$ con una pérdida de la reducción coronal de $2.8^{\circ}$, sin pérdida en la deformidad en cifosis.

Suk, et al. ${ }^{5}$ reportaron sus resultados con un seguimiento mínimo de 5 años, con una pérdida de la corrección coronal del 3\%. Di Silvestre, et al. ${ }^{19}$ compararon la instrumentación con tornillos transpediculares con la instrumentación híbrida, con un seguimiento de 6.7 años, y encontraron un mejor mantenimiento de la corrección de la deformidad mediante el uso de tornillos transpediculares y barras. Observaron un pérdida de $11.3^{\circ}$ de la corrección con el uso de instrumentaciones híbridas, contra $1.9^{\circ}$ de pérdida de la corrección utilizando tornillos transpediculares. De esta manera se justifica la superioridad biomecánica a largo plazo del uso de barras y tornillos transpediculares ${ }^{19}$.

Se realizó un seguimiento del estado clínico del paciente mediante la escala SRS-22, encontrando un resultado del $75 \%$ global; Lehman, et al. ${ }^{3}$, en una serie de 114 pacientes, reportaron un resultado global del 83.6\% ${ }^{3}$. En 2004, Bago, et al. ${ }^{20}$ realizaron un estudio de validación de la traducción y una validación cultural del cuestionario SRS-22, en el que se incluyó a 175 pacientes (152 mujeres y 23 hombres), probando 
que la versión en español del cuestionario SRS-22 es válida, con una excelente reproducción de todos sus rubros, y es equivalente y ajustable para la investigación clínica (Fig. 3).

En 2007, Rosales-Olivarez, et al. ${ }^{21}$ realizaron un estudio descriptivo del registro histórico del Instituto Nacional de Rehabilitación (INR), en el que incluyeron a 120 pacientes ( 45 hombres y 75 mujeres) con diagnósticos de escoliosis congénita, escoliosis idiopática infantil, escoliosis idiopática juvenil y EIA (25\%), con un promedio de edad de 12 años, tratados mediante abordaje posterior (54\%), anterior y mixto, mediante las técnicas mixtas con instrumentación tipo Luque I, II y III (63\%). En el estudio citado se describe una curva prequirúrgica de $55.58^{\circ}$, mientras que en la muestra analizada obtuvimos una curva inicial de $60.8^{\circ}$; en el estudio previo se reportó una corrección promedio de $25.92 \%$ en coronal, esto representa un $38.8 \%$ menos que la corrección evaluada en nuestro estudio actual (64\%). Tuvieron una tasa de complicación del 17\%, contra una tasa reportada en este estudio del $24 \%$, quizá por la mayor traslación al momento de la corrección de la curva (14.47 vs. $\left.39.12^{\circ}\right)$, así como por la gravedad de los casos, además de que el uso de tornillos transpediculares per se implica un mayor volumen de complicaciones; sin embargo, se reportó una incidencia de lesión medular del $5 \%$, similar al $4 \%$ de nuestro reporte.

El uso actual de tornillos transpediculares en el INR para la corrección de la deformidad vertebral ofrece mayor ventaja biomecánica sobre el uso de instrumentación mixta utilizada anteriormente. Al instrumentar las tres columnas anatómicas de la vértebra se corrige y mantiene la corrección de la deformidad con mayor efectividad que con las instrumentaciones mixtas, disminuyendo la tasa de complicaciones, el sangrado y el número de niveles instrumentados ${ }^{5}$.

\section{Conclusiones}

- El tratamiento de la EIA con tornillos transpediculares y barras es seguro y efectivo para corregir y mantener la deformidad escoliótica.

- El uso actual de tornillos transpediculares en nuestro instituto ofrece ventajas sobre el uso de instrumentación híbrida utilizada anteriormente para la corrección de la deformidad vertebral en la EIA, ofreciendo una mayor ventaja biomecánica y disminuyendo la tasa de complicaciones, el sangrado y el número de niveles instrumentados.

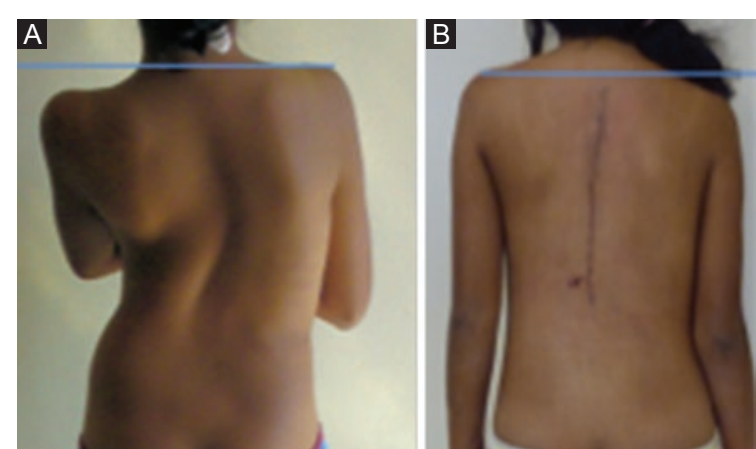

Figura 3. Imágenes clínicas, prequirúrgica $(\boldsymbol{A})$ y posquirúrgica (B), de una paciente sometida a manejo con tornillos transpediculares $y$ barras. Se observan corrección de la deformidad, adecuada alineación coronal y simetría de hombros y caderas.

\section{Financiación}

Los autores declaran carecer de fuente de financiación.

\section{Conflicto de intereses}

Los autores declaran que no existen conflictos de intereses.

\section{Responsabilidades éticas}

Protección de personas y animales. Los autores declaran que para esta investigación no se han realizado experimentos en seres humanos ni en animales.

Confidencialidad de los datos. Los autores declaran que han seguido los protocolos de su centro de trabajo sobre la publicación de datos de pacientes.

Derecho a la privacidad y consentimiento informado. Los autores han obtenido el consentimiento informado de los pacientes y/o sujetos referidos en el artículo. Este documento obra en poder del autor de correspondencia.

\section{Bibliografía}

1. Hwang SW, Samdani AF, Marks M, Bastrom T, Garg H, Lonner B, et al. Five-year clinical and radiographic outcomes using pedicle screw only constructs in the treatment of adolescent idiopathic scoliosis. Eur Spine J. 2013;22:1292-9.

2. Westrick ER, Ward WT. Adolescent idiopathic scoliosis: 5-year to 20-year evidence-based surgical results. J Pediatr Orthop. 2011;31(1 Suppl):S61-8.

3. Lehman RA Jr, Lenke LG, Keeler KA, Kim YJ, Buchowski JM, Cheh G, et al. Operative treatment of adolescent idiopathic scoliosis with posterior pedicle screw-only constructs: minimum three-year follow-up of one hundred fourteen cases. Spine (Phila Pa 1976). 2008;33:1598-604.

4. Kuklo TR, Potter BK, Lenke LG. Vertebral rotation and thoracic torsion in adolescent idiopathic scoliosis: what is the best radiographic correlate? J Spinal Disord Tech. 2005;18:139-47.

5. Suk SI, Lee SM, Chung ER, Kim JH, Kim SS. Selective thoracic fusion with segmental pedicle screw fixation in the treatment of thoracic idiopathic scoliosis: more than 5-year follow-up. Spine (Phila Pa 1976). 2005;30:1602-9. 
6. Danielsson AJ, Nachemson AL. Back pain and function 23 years after fusion for adolescent idiopathic scoliosis: a case-control study-part II. Spine (Phila Pa 1976). 2003;28:E373-83.

7. Kim YJ, Lenke LG, Kim J, Bridwell KH, Cho SK, Cheh G, et al. Comparative analysis of pedicle screw versus hybrid instrumentation in posterio spinal fusion of adolescent idiopathic scoliosis. Spine (Phila Pa 1976). 2006;31:291-8.

8. Asher MA, Burton DC. Adolescent idiopathic scoliosis: natural history and long-term treatment effects. Scoliosis. 2006;1:2.

9. Cochran T, Irstam L, Nachemson A. Long-term anatomic and functional changes in patients with adolescent idiopathic scoliosis treated by $\mathrm{Ha}$ rrington rod fusion. Spine (Phila Pa 1976). 1983;8:576-84.

10. Boos N, Aebi M. Spinal disorders. Fundamentals of diagnosis and treatment. En: Gruber P, Boeni T, editores. History of spinal disorders. Berlin Heidelberg: Springer-Verlag; 2008. p.29-37.

11. Dobbs MB, Lenke LG, Kim YJ, Kamath G, Peelle MW, Bridwell KH. Selective posterior thoracic fusions for adolescent idiopathic scoliosis: comparison of hooks versus pedicle screws. Spine (Phila Pa 1976). 2006;31:2400-4.

12. Ecker ML, Betz RR, Trent PS, Mahboubi S, Mesgarzadeh M, Bonakdapour A, et al. Computer tomography evaluation of Cotrel-Dubousse instrumentation in idiopathic scoliosis. Spine (Phila $\mathrm{Pa}$ 1976). 1988;13:1141-4.

13. Suk SI, Lee SM, Chung ER, Kim JH, Kim WJ, Sohn HM. Determination of distal fusion level with segmental pedicle screw fixation in single thoracic idiopathic scoliosis. Spine (Phila Pa 1976). 2003;28:484-91.
14. Abul-Kasim K, Ohlin A, Strömbeck A, Maly P, Sundgren PC. Radiological and clinical outcome of screw placement in adolescent idiopathic scoliosis: evaluation with low-dose computed tomography. Eur Spine J. 2010;19:96-104.

15. Gertzbein SD, Robbins SE. Accuracy of pedicular screw placement in vivo. Spine (Phila Pa 1976). 1990;15:11-4.

16. Belmont PJ Jr, Klemme WR, Dhawan A, Polly DW Jr. In vivo accuracy of thoracic pedicle screws. Spine (Phila Pa 1976). 2001;26:2340-6.

17. Suk SI, Lee CK, Kim WJ, Chung YJ, Park YB. Segmental pedicle screw fixation in the treatment of thoracic idiopathic scoliosis. Spine (Phila $\mathrm{Pa}$ 1976). 1995;20:1399-405.

18. Kim YJ, Bridwell KH, Lenke LG, Kim J, Cho SK. Proximal junctional kyphosis in adolescent idiopathic scoliosis following segmental posterior spinal instrumentation and fusion: minimum 5-year follow-up. Spine (Phila Pa 1976). 2005:30:2045-50.

19. Di Silvestre M, Bakaloudis G, Lolli F, Vommaro F, Martikos K, Parisini P. Posterior fusion only for thoracic adolescent idiopathic scoliosis of more than 80 degrees: pedicle screws versus hybrid instrumentation. Eur Spine J. 2008;17:1336-49.

20. Bago J, Climent JM, Ey A, Pérez-Grueso FJ, Izquierdo E. The Spanish version of the SRS-22 patient questionnaire for idiopathic scoliosis: transcultural adaptation and reliability analysis. Spine (Phila Pa 1976). 2004;29:1676-80.

21. Rosales-Olivarez LM, García J, Miramontes-Martínez VP, Alpízar-Aguirre A, Arenas-Sordo M de L, Reyes-Sánchez AA. Tratamiento quirúrgico de la escoliosis. Control de evolución mínimo de 5 años. Cir Cir. 2007;75:93-7. 